\title{
Da Aplysia à Constituição: Evolução de Conceitos na Análise do Comportamento
}

\author{
João Claudio Todorov ${ }^{12}$ \\ Universidade Católica de Goiás
}

\begin{abstract}
Resumo
Objetivou-se apresentar a evolução de conceitos na análise do comportamento, especialmente nos trabalhos de Skinner, desde a definição do conceito de operante, de contingência de reforço, até os processos de seleção por conseqüências, seguindo o desenvolvimento de uma linguagem teórica que abarca desde o comportamento de moluscos até o texto da Constituição, enquanto metacontingência. Os trabalhos iniciais de Skinner usavam a linguagem desenvolvida por Pavlov no estudo de reflexos condicionados para abranger todo o comportamento. De sua dissertação de doutorado até os trabalhos publicados nos anos 1980, Skinner evoluiu e com ele toda a análise do comportamento, que hoje usa a mesma linguagem mais sofisticada para abordar temas complexos como práticas culturais e a sobrevivência de culturas.
\end{abstract}

Palavras-chave: Operante; contingência de reforço; seleção por conseqüências; metacontingência; análise do comportamento.

From Aplysia to the Constitution: Evolution of Concepts in Behavior Analysis

\begin{abstract}
This work presents the evolution of concepts in behavior analysis, especially in the writings of Skinner, from the definition of operant behavior and reinforcement contingency to processes of selection by consequences, following the development of a conceptual language that covers both the behavior of molluscs and the metacontingencies included in the constitution of a country. Skinner's first papers used the terminology developed by Pavlov in his studies of conditioned reflexes to deal with behavior in general. From Skinner's doctoral dissertation to the papers published in the 80 's there was an evolution, followed by the field of behavior analysis, which today uses the same, sophisticated language to deal with complex issues like cultural practices and the survival of cultures.

Keywords: Operant; reinforcement contingency; selection by consequences; metacontingency; behavior analysis.
\end{abstract}

Nosso tema começa com o reflexo. No início do século XX o trabalho de Pavlov colocou em evidência um conceito que veio a marcar a história da psicologia. No reflexo cada estímulo estava ligado a cada resposta pelo sistema nervoso. Comportamentos complexos eram vistos como cadeias de reflexos, intrincadas interconexões de neurônios. Os estudos iniciais de Pavlov exploravam o papel do ambiente como instigador de respostas adrede preparadas para aquela espécie por uma herança genética, resultado de milhões de anos de interações de seus antepassados com ambientes variáveis. O trabalho de experimentação revelou logo seus limites. $\mathrm{Na}$ aprendizagem, na aquisição de novos reflexos, o novo era a ampliação de aspectos do ambiente com poder de eliciar a resposta, mas a resposta em si não mudava. Um som previamente neutro passa a eliciar salivação, anteriormente provocada apenas pelo alimento. O repertório do indivíduo foi aumentado, na medida em que podemos

\footnotetext{
${ }^{1}$ Trabalho apresentado na $54^{a}$ Reunião Anual da Sociedade Brasileira para o Progresso da Ciência, Goiânia, Goiás, julho de 2002.

${ }^{2}$ Endereço para correspondência: SHIN QI 01 Conj. 09 Casa 11, 71505 090, Brasília, DF.E-mail: todorov@unb.br
}

falar agora de dois reflexos, um incondicionado, ou inato, outro condicionado, ou aprendido; a salivação, contudo, é a mesma.

Reflexos tratam da economia interna do organismo. Nenhum outro é tão simples, ou pelo menos tão fácil de ser estudado, quanto o da salivação. Todos, porém, tratam da adaptação do organismo, da adaptação de seu funcionamento enquanto ser biológico, a mudanças no ambiente.

O que hoje chamamos de análise do comportamento começou com o estudo do que Skinner denominou "reflexo alimentar". O que parecia ser um simples reflexo a ser investigado para uma tese de doutorado mostrou-se uma fonte inesperada de novos conhecimentos (Skinner, 1930, 1931). Ratos eram colocados em uma pequena câmara experimental com acesso a pelotas de alimento em uma cuba colocada em uma das paredes. Com peso corporal controlado e número de horas de privação de alimentos constante, Skinner registrava o número de pelotas consumidas por unidade de tempo. Um equipamento rudimentar produzia um gráfico on-line da freqüência acumulada de pelotas consumidas, usando um quimógrafo adaptado. O registro do quimógrafo mostrava visualmente 
a diminuição na força do reflexo alimentar (número de pelotas por minuto) como função do número de pelotas consumidas. Para automatizar esse registro Skinner colocou uma portinhola basculante fechando a boca da cuba. Os ratos empurravam a porta para chegar até o alimento, o que acionava um mecanismo que registrava automaticamente $\mathrm{o}$ consumo de uma pelota. Na verdade, registrava o empurrão na porta, mas não garantia que apenas uma pelota era consumida cada vez que o rato tinha acesso ao alimento. $O$ passo seguinte foi esvaziar a cuba e construir um mecanismo dispensador de alimento. Uma pequena barra de metal, quando pressionada, fazia cair na cuba uma pelota de alimento por vez. Agora, o contador eletromecânico acionado pela pressão à barra de metal registrava fidedignamente o consumo de cada pelota. $\mathrm{O}$ aparato foi um sucesso e veio a ser o precursor das milhões de Caixas de Skinner (as Skinner Boxes) fabricadas nos últimos 70 anos. Contudo, havia um porém. Skinner produzira uma situação com dois reflexos encadeados. Um já era conhecido: a visão do alimento, estímulo incondicionado, eliciava a ingestão do alimento, resposta incondicionada. E a pressão à barra? Que estímulo eliciava a pressão à barra? A visão da barra?

Se tivesse começado seus estudos por qualquer outro reflexo seu trabalho seria bem mais complicado. E no entanto, o modelo simples de reflexo desenvolvido a partir da salivação em cães foi extensivamente usado em tentativas de explicar todo o comportamento. $\mathrm{Na}$ ausência de um estímulo eliciador incondicionado conhecido, postulava-se a existência de algum. Skinner prosseguiu por algum tempo com a explicação de que a visão da barra de metal eliciava o movimento de pressioná-la para baixo.

Por essa época os trabalhos de Thorndike com gatos em sua caixa-problema, que levaram à formulação da Lei do Efeito, já tinham 20 anos. A lei era muito conhecida na psicologia aplicada, especialmente à educação, mas seu possível relacionamento com os trabalhos de Pavlov não havia sido sistematicamente explorado (Catania, 1999; Chance, 1999; Nevin, 1999). Um primeiro passo nessa direção foi dado por Skinner em 1935, reconhecendo a existência de dois processos diferentes, dois tipos de condicionamento (Skinner, 1935). A psicologia soviética ficou muito mais tempo presa ao conceito de arco-reflexo de Pavlov, sem reconhecer a importância das conseqüências do comportamento (Anokhin, citado em Skinner, 1969).

A necessidade da distinção operante-respondente é claramente exposta na resposta a Konorski e Miller (Skinner, 1937). O comportamento respondente trata da economia interna do organismo. A evolução da espécie dotou o indivíduo com um conjunto de reações a estímulos do ambiente, e o que a aprendizagem faz é aumentar o leque de estímulos que podem controlar uma determinada resposta. O comportamento operante engloba as operações do organismo sobre o ambiente, alterando esse ambiente, e tais alterações podem determinar a probabilidade futura dessas ações.

Nesse sentido, o comportamento de um indivíduo hoje é resultado de dois processos semelhantes de seleção por conseqüências. Para a espécie, ao longo da evolução, as conseqüências de seus atos selecionaram indivíduos com certas características, que geraram descendência. A história da espécie é uma história de sobrevivência por adaptação a mudanças no ambiente. Para o indivíduo, sua constituição genética determina seu repertório incondicionado, e as conseqüências de suas ações sobre o ambiente determinam (selecionam) quais respostas serão repetidas no futuro. A história do indivíduo parte da história da espécie. Muito além da fantástica variabilidade no seu patrimônio genético, cada indivíduo age sobre seu ambiente e sua ação é modificada por suas conseqüências sobre o ambiente. $\mathrm{O}$ que é no nascimento um repertório predominantemente reflexo, com padrões de ação fixos e característicos de cada espécie, amplia-se com o desenvolvimento do repertório operante. Em certos casos o comportamento operante evolve diretamente de um respondente, como o sugar o seio materno nos mamíferos. No nascimento o sugar é basicamente reflexo, e pode ser eliciado por um toque nos lábios do bebê. Rapidamente passa para o controle operante e tem todas as características das respostas mantidas por suas conseqüências.

Voltando ao protótipo da caixa de Skinner: ao sair de uma situação em que consumia pelotas de alimento colocadas à sua disposição, sem restrições, para outra na qual era preciso pressionar uma barra para que uma pelota aparecesse, o animal saiu de uma situação experimental planejada para estudar reflexos para outra, com todas as características da caixa-problema de Thorndike, mas com uma vantagem: o rato podia repetir a resposta sem a interferência do experimentador. $\mathrm{Na}$ gaiola problema, ou nos labirintos, a repetição do ato depende do experimentador. Para repetir uma tentativa o animal deve ser manualmente colocado na gaiola ou no início do labirinto. A freqüência de repetição não pode ser uma variável dependente. Skinner descobriu como usar freqüência da resposta como variável dependente em estudos do comportamento instrumental.

Para entender a rapidez de novos desenvolvimentos a partir desses eventos metodológico-conceituais é preciso olhar para a formação acadêmica de Skinner. Depois de um curso de graduação em literatura inglesa, interesse que vai acompanhálo pelo resto da vida, Skinner dedica-se com afinco a duas outras áreas: filosofia da ciência e biologia. Bertrand Russell, Ernst Mach, por um lado, e expoentes da biologia em Harvard, por outro, marcam o início de sua formação científica. É 
introduzido ao behaviorismo de Watson por um colega de pósgraduação, Fred S. Keller (em 1953 dedica um livro a Keller, escrevendo de próprio punho o agradecimento por representar a única brisa de behaviorismo na atmosfera de Harvard - o próprio Skinner se encarregaria anos mais tarde de transformar essa brisa em vendaval). A partir da distinção operanterespondente, Skinner usa sua formação para o desenvolvimento de um sistema, apresentado em livro ainda na década de 1930 (Skinner, 1938), com o título nada modesto de "O Comportamento dos Organismos". Rearranja termos e conceitos da psicologia experimental para mostrar, na probabilidade de ocorrência de uma resposta do rato albino, instâncias de aprendizagem, motivação, percepção, entre outros. Ao mesmo tempo, e ao que parece independentemente, começa a trabalhar com um comportamento especificamente humano, a linguagem. Em 1936 publica o que seria para a audição o que o teste de Rorschach é para a visão: O "Somador Verbal" (um tipo de gravador gerava ruídos desconexos; ouvindo-os o sujeito deveria dizer que palavras estavam sendo ditas). A partir daí trabalha paralelamente em duas obras: "Ciência e Comportamento Humano” (Skinner, 1953) e “OComportamento Verbal”' (Skinner, 1957). As potencialidades da investigação do comportamento na caixa de Skinner também são exploradas paralelamente com a ajuda de Charles B. Ferster e publicadas em Schedules of Reinforcement (Ferster \& Skinner, 1957).

É interessante notar que nos anos 1950 Skinner aprofunda seu trabalho de análise experimental do comportamento animal em um livro (Ferster \& Skinner, 1957), dedica outro livro todo a um comportamento especificamente humano, o comportamento verbal (Skinner, 1957), e amplia a abrangência de seu livro inicial de 1938 (Skinner, 1938) para mostrar em "Ciência e Comportamento Humano" (Skinner, 1953) que havia desenvolvido uma linguagem conceitual que poderia abarcar tudo aquilo quer poderia ser chamado de psicologia, inclusive a psicanálise. Citar os títulos de alguns capítulos pode ser uma maneira de dar exemplos do que acabamos de afirmar: "Autocontrole”, cap. 15; “Pensamento", cap. 16; "Eventos privados...", cap. 17; "O self”, cap. 18; "Psicoterapia”, cap. 24. Neles são analisados conceitos que até hoje ainda são vistos pelos menos avisados como incompatíveis com uma análise do comportamento: id, ego, superego, autoconhecimento, repressão, sublimação, identificação, projeção, atos falhos, entre outros.

Com "Ciência e Comportamento Humano" (Skinner, 1953) Skinner abre uma terceira visão do processo de seleção pelas conseqüências. Vimos que para as espécies o processo resulta na sobrevivência de indivíduos com determinadas características. Para o indivíduo a seleção de respostas por suas conseqüências modela um repertório comportamental a partir das possibilidades dispostas pela herança genética e das regras da sociedade à qual pertence. Em 1953 Skinner começa a analisar como um processo semelhante atua na evolução das culturas:

... o indivíduo adquire do grupo um extenso repertório de usos e costumes. O que o homem come e bebe, e como o faz, os tipos de comportamento sexual em que se empenha, como constrói uma casa, ou desenha um quadro, rema um barco, os assuntos sobre os quais fala ou cala, a música que compõe, os tipos de relações pessoais que tem, e os tipos que evita - tudo depende em parte dos procedimentos do grupo de que é membro. Os usos e costumes vigentes em muitos grupos, é claro, têm sido extensamente descritos por sociólogos e antropólogos. Aqui nos preocuparemos apenas com as espécies de processos que exemplificam. (Skinner, 1953, p. 415)

Mais de um terço do livro é dedicado a temas que até então não eram sistematicamente abordados pela psicologia; na divisão das ciências, pertenciam à antropologia, ciência política, direito, economia, sociologia, etc. Skinner não respeita essas fronteiras na busca de um comportamento desgarrado: o psicólogo dever ir aonde o comportamento está. Se vivesse hoje Skinner assim se manifestaria sobre o comportamento recente dos argentinos:

Muitas generalizações ao nível do grupo não precisam de modo algum se referirem ao comportamento. Há uma antiga lei em economia, chamada Lei de Gresham, que afirma que a moeda má tira de circulação a boa moeda. Se pudermos concordar a respeito do que seja moeda, boa ou má, e quando está circulando, poderemos expressar esse princípio geral sem referência específica ao uso da moeda por indivíduos. Encontram-se generalizações semelhantes na sociologia, na antropologia cultural, na lingüística e na história. Mas uma "lei social" deve ser gerada comporta com o mesmo corpo e de acordo com os mesmos processos usados em uma situação não social. Se o indivíduo que possui pelo comportamento de indivíduos. É sempre o indivíduo que se comporta, e que se duas moedas, uma boa e outra má, tende a gastar a má e guardar a boa - uma tendência que pode ser explicada em termos de contingências de reforço - e se é válido para um grande número de pessoas, surge o fenômeno descrito pela Lei de Gresham. O comportamento do indivíduo explica o fenômeno do grupo. (Skinner, 1953, p. 171)

Não se trata, pois, de apenas traduzir termos de outra abordagem teórica para uma linguagem diferente. Skinner aponta a necessidade e a viabilidade de se analisar as variáveis envolvidas em situações para as quais, por exemplo, Freud desenvolveu os conceitos de id, ego e superego: 
Freud concebia o ego, superego e o id como agentes distintos dentro do organismo. O id era responsável pelo comportamento que em última instância fosse reforçado com alimento, água, contato sexual, e outros reforçadores biológicos primários. Não era muito diferente do Adão da teologia judaico-cristã, egoísta e agressivo, preocupado com as privações básicas e indiferente às necessidades semelhantes por parte dos outros. $\mathrm{O}$ superego $-\mathrm{a}$ "consciência" da teologia judaico-cristã - era responsável pelo comportamento que controlava o id. Usava técnicas de autocontrole adquiridas do grupo. Quando estas eram verbais constituíam a "vOz da consciência". O superego e o id opunhamse inevitavelmente um ao outro, eFreud concebia-os quase sempre como em conflito violento. Apelou ainda para um terceiro agente - o ego - que além de tentar alcançar um acordo entre o id e o superego, também lidava com as exigências práticas do ambiente. Podemos discutir qualquer análise que apele para um eu ou uma personalidade como um determinante interior da ação, mas os fatos que foram representados por estes estratagemas não podem ser ignorados. Os três eus ou personalidades do esquema freudiano representam características importantes do comportamento em um meio social. (Skinner, 1953, p. 284-285)

"Ciência e Comportamento Humano" foi um marco no desenvolvimento da análise comportamental aplicada. Foram sem conta os caminhos sugeridos por Skinner e suas análises aplicáveis a quaisquer situações envolvendo seres humanos em interação. Mas o trabalho ainda estava incompleto. O arcabouço teórico montado por Skinner evoluiu aos poucos (Sério, 1983, 1990) e continua sendo aperfeiçoado (Ex.: Critchfield \& Kollins, 2001; Davison \& Nevin, 1999; Mace, 1996; Michael, 2000; Nevin, 1996; Sidman, 2000; Todorov, 2002; Vollmer \& Hackenberg, 2001; Williams, 1988; Zentall \& Smeets, 1996).

Nos anos 1960 o conceito de contingências de reforço começa a ganhar importância maior, e o processo de seleção por conseqüências tem grande relevo (Skinner, 1966, 1969, 1975, 1977, 1981, 1984). Nessas publicações Skinner aperfeiçoa o conceito de seleção por conseqüências, incluindo uma terceira fonte: a cultura. Ao analisar o comportamento humano mostrou as maneiras pelas quais o comportamento do indivíduo é aprendido e mantido pelas conseqüências sociais apresentadas por outros indivíduos. Em oposição às contingências naturais (Ex.: aproximar a mão do fogo é punido com a dor da queimadura), as contingências sociais são muitas vezes arbitrárias. Como se estabelecem e se mantêm essas contingências?

Skinner chega necessariamente a essa questão por seu interesse persistente em desenvolver conhecimento útil. $\mathrm{O}$ desenvolvimento de uma análise comportamental aplicada como meta é evidente em Skinner e seus colaboradores (Ayllon \& Azrin, 1968; Ayllon \& Michael, 1959; Holland, 1958; Keller \& Schoenfeld, 1950). Se são as contingências sociais que modelam o comportamento dos indivíduos, o que modela uma contingência social? Essa é uma pergunta que não pode ser respondida no laboratório. Por outro lado, os dados estão disponíveis nas ciências sociais, e Skinner faz amplo uso deles. Não se trata, pois de ignorar as ciências sociais ou de negar sua importância e substituí-las por uma nova disciplina científica. Os mesmos métodos de análise funcional utilizados para estudar o comportamento de indivíduos são usados para pensar as informações que nos oferece a antropologia, por exemplo. Em Contingencies of Reinforcement (Skinner, 1969), Skinner usa um exemplo hipotético de alguém que teria a tarefa de planejar uma cultura para mostrar como e porque as práticas culturais persistem:

Uma comunidade é uma entidade, com vida própria. Irá sobreviver ou perecer, e o planejador deve manter isso em mente. O problema é que a sobrevivência muitas vezes é facilitada por comportamentos que não apenas não são reforçados, mas que também podem ter conseqüências punitivas (ou mesmo letais). Contingências filogenéticas de sobrevivência fornecem exemplos. Quando um membro de uma manada de animais que está pastando percebe a aproximação de um predador e solta um grito de alerta, seu comportamento aumenta a probabilidade do grupo escapar e sobreviver, mas o membro que grita o alerta chama a atenção do predador para si e pode perecer. Contingências ontogenéticas de reforço funcionam da mesma maneira: a cultura induz o herói a morrer por seu país ou o mártir por sua religião. (Skinner, 1969, p. 40)

Por quê uma cultura iria se preocupar com sua sobrevivência? Sobreviver para quê? Como sabemos que uma cultura está evoluindo para a direção certa? Questões como essas mostram um mal-entendido a respeito da natureza da evolução, biológica e cultural. Os processos de mutação e seleção não requerem, e podem não fornecer, qualquer projeto prévio do estado para o qual conduzem. (Skinner, 1969, p. 41)

O antílope desgarrado não grita para alertar o grupo da proximidade do leão. Grita de medo sem ter tido que aprender que o leão é perigoso. As contingências filogenéticas foram responsáveis pela maior probabilidade de sobrevivência de grupos de antílopes mais assustadiços; o primeiro a gritar de medo atrai a atenção do predador. Enquanto o leão se ocupa de uma presa, o bando tem tempo de fugir. A conseqüência do comportamento de um indivíduo tem valor de sobrevivência para o grupo, mas a explicação não depende 
da hipótese de um plano adrede preparado pela natureza para garantir a sobrevivência do grupo.

O triste evento da destruição das Torres Gêmeas de Nova Iorque por terroristas suicidas, provocando mais de 3000 mortes, e os atuais atentados suicidas em Israel, mostram a força que a orientação do grupo, no caso o grupo religioso, tem sobre o comportamento do indivíduo. De novo, a conseqüência do ato individual não está necessariamente ligada à sobrevivência da cultura; para entender $\mathrm{O}$ comportamento dos suicidas é preciso entender o desenvolvimento do conceito de jïhad, a guerra santa, na cultura islâmica: o que garante o comportamento do indivíduo são as promessas de conseqüências para o comportamento do próprio suicida.

Setenta anos depois da tese de doutorado de Skinner os frutos de seu trabalho estão por toda parte, seja nos estudos dos batimentos cardíacos da Aplysia (Todorov, 1991), seja na evolução do conceito de contingência para abarcar eventos sociais que envolvem todo um país (Todorov, 1987). A importância atual da análise comportamental aplicada pode ser facilmente constatada (Ex.: Austin \& Carr, 2000; Biglan, 1995; Guerin, 1994; Hayes, Jacobson, Folette \& Dougher, 1994; Kohlenberg \& Tsai, 1991; Leslie \& Blackman, 2000). Sua visão de homem e de mundo é cada vez mais um alerta necessário: somos responsáveis por nosso futuro, um porvir glorioso não está escrito nas estrelas:

O homem é em grande parte responsável pelo ambiente em que vive. Mudou o mundo físico para minimizar as propriedades aversivas e maximizar os reforços positivos, e construiu sistemas de governo, religião, educação, econômicos e psicoterápicos que promovem contatos pessoais satisfatórios e o tornam mais habilidoso, informado, produtivo, e feliz. Ele está engajado em um exercício gigantesco de auto-controle, e como resultado tem cada vez mais tornado real seu potencial genético. (Skinner, 1969, p. 45)

\section{Referências}

Austin, J. \& Carr, J. (2000). The handbook of applied behavior analysis. Reno: Context Press.

Ayllon, T. \& Azrin, N. H. (1968). The token economy. New York: Appleton-CenturyCrofts.

Ayllon, T. \& Michael, J. (1959). The psychiatric nurse as a behavioral engineer. Journal of the Experimental Analysis of Behavior, 2, 323-334.

Biglan, A. (1995). Changing cultural practices: A contextualist framework. for intervention research. Reno: Context Press.

Catania, A. C. (1999). Thorndike's legacy: Learning, selection, and the law of effect. Journal of the Experimental Analysis of Behavior, 72, 425-428.
Chance, P. (1999). Thorndike's puzzle boxes and the origins of the experimental analysis of behavior. Journal of the Experimental Analysis of Behavior, 72, 433440.

Critchfield, T. S. \& Kollins, S. H. (2001). Temporal discounting: Basic research and the analysis of socially important behavior. Journal of Applied Behavior Analysis, 34, 101-122.

Davison, M. \& Nevin, J. A. (1999). Stimuli, reinforcers, and behavior: An integration. Journal of the Experimental Analysis of Behavior, 71, 439-482.

Ferster, C. B. \& Skinner, B.F. (1957). Schedules of reinforcement. New York: AppletonCentury-Crofts.

Guerin, B. (1994). Analyzing social behavior: Behavior anabsis and the social sciences. Reno: Context Press.

Hayes, S. C., Jacobson, N. S., Folette, M. V. \& Dougher, M. (1994). Acceptance and change: Content and context in psychotherapy. Reno: Context Press.

Holland, J. G. (1958). Human vigilance. Science, 128, 61-67.

Keller, F. S. \& Schoenfeld, W. N. (1950). Principles of psychology. New York: AppletonCentury-Crofts.

Kohlenberg, R. J. \& Tsai, M. (1991). Functional analytic psychotherapy: Creating intense and curative therapeutic relationships. New York: Plenum Press.

Leslie, J. \& Blackman, D. (2000). Experimental and applied analysis of human behavior. Reno: Context Press.

Mace, F. C. (1996). In pursuit of general behavior relations. Journal of Applied Behavior Analysis, 29, 557-563.

Michael, J. (2000). Implications and refinement of the establishing operation concept. Journal of Applied Behavioral Analysis, 33, 401-410.

Nevin, J. (1996). The momentum of compliance. Journal of Applied Behavior Analysis, 29, 535-547.

Nevin, J. (1999). Analyzing Thorndike's law of effect: The question of stimulusresponse bonds. Journal of the Experimental Analysis of Behavior, 72, 447-450.

Sério, T. M. A. P. (1983). A noşão de classe de respostas operante: Sua formulação inicial. Dissertação de Mestrado não-publicada, Departamento de Psicologia Experimental, Universidade de São Paulo. São Paulo, SP.

Sério, T. M. A. P. (1990). Um caso na história do método científico: Do reflexo ao operante. Tese de Doutorado não-publicada, Programa de Estudos Pós-Graduados em Psicologia Social, Pontifícia Universidade Católica de São Paulo. São Paulo, SP.

Sidman, M. (2000). Equivalence relations and the reinforcement contingency. Journal of the Experimental Analysis of Behavior, 74, 127-146.

Skinner, B. F. (1930). On the conditions for elicitation of certain eating reflexes. Proceedings of the National Academy of Sciences, 16, 433-438.

Skinner, B. F. (1931). The concept of reflex in the description of behavior. Journal of General Psychology, 5, 427-458.

Skinner, B. F. (1935). Two types of conditioned reflex and a pseudotype. Journal of General Psychology, 12, 66-77.

Skinner, B. F. (1936). The verbal summator and a method for the study of latent speech. Journal of Psychology, 2, 71-107.

Skinner, B. F. (1937).

Skinner, B. F. (1938). The behavior of organisms. New York: Appleton-CenturyCrofts.

Skinner, B. F. (1953). Science and human behavior. New York: MacMillan.

Skinner, B. F. (1957/1978). Verbal behavior. New York: Appleton-Century-Crofts.

Skinner, B. F. (1966). The phylogeny and ontogeny of behavior. Science, 153, 12041213.

Skinner, B. F. (1969). Contingencies of reinforcement. New York: Appleton-CenturyCrofts.

Skinner, B. F. (1975). The shaping of phylogenic behavior. Journal of the Experimental Analysis of Behavior, 24, 117-120.

Skinner, B. F. (1977). Herrnstein and the evolution of behaviorism. American Psychologist, 32, 1006-1012.

Skinner, B. F. (1981). Selection by consequences. Science, 213, 501-504. 
Skinner, B. F. (1984). The evolution of behavior. Journal of the Experimental Analysis of Behavior, 41, 217-221.

Todorov, J. C. (1987). A constituição como metacontingência. Psicologia: Ciência e Profissão, 7, 9-13.

Todorov, J. C. (1991). Progressos no estudo das bases neurais da aprendizagem. Psicologia: Teoria e Pesquisa, 7, 303-310.

Todorov, J. C. (2002). Evolução do conceito de operante. Psicologia: Teoria e Pesquisa, $18,123-127$.

Vollmer, T. R. \& Hackenberg, T. D. (2001). Reinforcement contingencies and social reinforcement: Some reciprocal relations between basic and applied research. Journal of Applied Behavior Analysis, 34, 241-253.
Williams, B. A. (1988). Reinforcement, choice, and response strength. Em R. C. Atkinson, R. J. Herrnstein, G. Lindzey \& R. D. Luce (Orgs.), Stevens' bandbook of experimental psychology (Vol. 2, pp. 167-244). New York: Wiley.

Zentall, T. R. \& Smeets, P. M. (1996). Stimulus class formation in bumans and animals. New York: Elsevier.

Recebido: 28/01/2003

$1{ }^{a}$ Revisão: 22/04/2003

$2^{a}$ Revisão: 06/08/2003

Aceite Final: 12/08/2003

Sobre o autor

João Cláudio Todorov é Professor aposentado e colaborador da Universidade de Brasília. É

Professor da Universidade Católica de Goiás. 